\title{
Fiber fuse in chalcogenide photonic crystal fibers
}

\author{
Sida Xing, ${ }^{*}$ Svyatoslav Kharitonov, Jianqi Hu, and Camille-Sophie Brès \\ Ecole Polytechnique Fédérale de Lausanne (EPFL), Photonic Systems Laboratory, STI-IEL, Station 11, CH-1015 Lausanne, Switzerland \\ ${ }^{*}$ Corresponding author: si.xing@epfl.ch
}

Received 7 February 2018; revised 22 February 2018; accepted 25 February 2018; posted 26 February 2018 (Doc. ID 322502$)$; published 19 March 2018

\begin{abstract}
We observe fiber fuse in tapered GeAsSe photonic crystal fibers (PCF) at around $7 \mathrm{MW} / \mathrm{cm}^{2}$ of intra-core intensity. Vertically cleaved facets from the un-tapered regions and the tapered regions were imaged. The images show shallow voids of different shapes confined to the fiber core. After longitudinally polishing a segment of the PCF, we imaged the PCF internal structure's top view, revealing the fuse voids' geometries and periodicity. In addition, fiber fuse was terminated in one PCF sample by a fast laser shutdown, hence saving a small segment from catastrophic damage. Four-wave-mixing was performed on this transmissive segment to estimate the dispersion. The results yielded an evident hole-pitch ratio change after fiber fuse. To our knowledge, this is the first report of fiber fuse on non-silica glass fibers and the first study of its aftermath on this un-destroyed segment of PCFs. ( 2018 Optical Society of America
\end{abstract}

OCIS codes: (060.2290) Fiber materials; (060.2400) Fiber properties; (060.4005) Microstructured fibers; (140.3330) Laser damage; (350.5340) Photothermal effects; (190.4410) Nonlinear optics, parametric processes.

https://doi.org/10.1364/OL.43.001443

Fiber fuse-an optical discharge occurring within a fiber core due to thermal runaway at a localized defect-leads to irreversible and catastrophic damage to optical fiber systems. The induced plasma propagates backward towards the pump source, where energy is provided, and leaves isolated voids inside the fiber core. Since its first observation in the 1980s [1], studies have been performed on this topic with both continuous-wave (CW) lasers and pulsed lasers $[1,2]$. An intensity threshold was found to be in the order of several $\mathrm{MW} / \mathrm{cm}^{2}$ with a propagation speed in order of $\mathrm{m} / \mathrm{s}$ [2]. Due to its severe threat to dense wavelength division multiplexing systems, fiber amplifiers, and fiber laser systems, both characterization [3-6] and suppression/termination techniques [7-9] of fiber fuse, mostly limited to silica fiber networks, have been explored. For other fiber materials, fiber fuse in polymer fibers has also been observed in 2014 [10]. Another study performed in fluoride and $\mathrm{As}_{2} \mathrm{~S}_{3}$ fibers claimed fiber fuse to be improbable in such fibers due to their low melting temperatures [11]. Thus, for a long time, it was believed that the destruction mechanism inside soft-glass fibers was purely thermal and distinct from fiber fuse in silica fibers $[1,12]$.

In recent years, the interest in mid-infrared (MIR) applications and compact MIR platforms has catalyzed the development of MIR fibers with novel materials and structures, as well as improved transmissions [13]. Among them, chalcogenide glass $(\mathrm{ChG})$ fibers possess the largest transmission bands, they are nonlinear, and they can be fabricated into suspended core [14] or photonic crystal fiber (PCF) [15] geometries for various applications. It followed that great efforts have been put into ChG fiber development and optimization. In terms of ChG fiber platforms, breakthroughs in both CW and pulsed applications have been reported in the recent two years. For pulsed applications, an all-fiber MIR optical parametric oscillator [16], a parametric wavelength converter [17], and an allfiber MIR supercontinuum source [18] became feasible. For CW laser applications, advances in fabrication technology now allow for amplified parametric conversion in MIR [19], power delivery [20], and integration into the silica fiber network [21]. In CW applications, power intensities have exceeded $5 \mathrm{MW} / \mathrm{cm}^{2}$. Such values, orders of magnitude higher than what used to be believed feasible [13], are sufficient to ignite fiber fuse in silica fibers. At this early stage of research and development, it is therefore important to look back into the possibility of fiber fuse in ChG fibers. In addition, fiber fuse inside a different material platform can provide more insights and lead to better understanding of the phenomenon.

In this Letter, we report, to the best of our knowledge, the first observation of fiber fuse in non-silica glass fibers. Inside two GeAsSe tapered PCFs (referred to as T-PCF1 and TPCF2), fast backward propagating damages were observed, starting at a power intensity of $6.5 \mathrm{MW} / \mathrm{cm}^{2}$. The fiber fuse propagation speed is larger than $0.6 \mathrm{~m} / \mathrm{s}$. Inspection with a scanning electron microscope (SEM) and an optical microscope exhibited smooth, shallow, and discrete voids confined within the fiber core. We also polished a small segment from the untapered region along its longitudinal axis and revealed the quasi-periodic nature of the voids. Because of a quick shutdown of the pump laser, we managed to terminate fiber fuse in TPCF2 and save a $28-\mathrm{cm}$ segment out of the $1.2-\mathrm{m}$ taper waist from catastrophic damage. With a parametric conversion experiment, clear substantial change of dispersion was measured. We believe this is due to a decrease in the hole-pitch ratio of the 


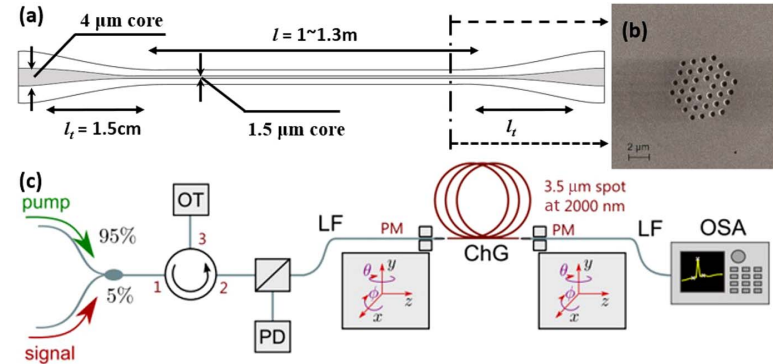

Fig. 1. (a) Geometry of the GeAsSe PCF (not to scale), (b) SEM image of a facet in taper waist, (c) laser setup for parametric conversion test. OT, optical terminator; PD, photodetector; LF, lensed fiber; OSA, optical spectrum analyzer; PM, polarization maintaining (fiber).

PCF, where the hole size shrinks due to the deposition of vaporized core material along the sidewalls. This first study of fiber fuse aftermath in PCF systems shows that the impact of the plasma propagation goes beyond the destroyed fiber sections and significantly changes PCF dispersion. Therefore, fiber fuse suppression in any dispersion-sensitive PCF system requires more parameters to be considered.

Our ChG PCFs were fabricated from a highly purified $\mathrm{Ge}_{10} \mathrm{As}_{22} \mathrm{Se}_{68}$ glass rod with three rings of air holes, which was provided by SelenOptics. The preform was drawn into a PCF with a $4-\mu \mathrm{m}$ core size and a $130-\mu \mathrm{m}$ outer diameter. The holepitch ratio was estimated at 0.58 . A small segment was tapered to a core size of approximately $1.5 \mu \mathrm{m}$. In this Letter, two tapers with waist lengths of $1 \mathrm{~m}$ (T-PCF1) and $1.2 \mathrm{~m}$ (T-PCF2), respectively, were tested. The transition region has a length of approximately $l_{t}=1.5 \mathrm{~cm}$. Such geometry leads to a nonlinear parameter $\gamma$ of $10(\mathrm{~W} \cdot \mathrm{m})^{-1}$, measured at a pump wavelength of 1950 nm. Figure 1(a) shows the PCF geometry and Fig. 1(b) shows an SEM image of the cleaved fiber waist facet.

The experimental setup [Fig. 1(c)] consists of two thuliumdoped fiber lasers serving as a signal and a pump, respectively. The pump laser cavity utilizes fiber Bragg grating (FBG) as a wavelength selective element, where 1950-nm, 1980-nm, 2008-nm, and 2040-nm wavelengths are available. The signal laser features continuous sweeping of lasing wavelength. The two lasers are combined by a 95/5 coupler and pass through a polarization beam splitter to ensure the same polarization. Two identically lensed fibers, fabricated on polarization maintaining fibers, couple the light into and out of the fiber under test. The input lensed fiber can rotate around its longitudinal axis for a birefringence check. The ChG PCF waist has a 0.5$\mathrm{dB} / \mathrm{m}$ measured propagation loss at $1.95 \mu \mathrm{m}$. More details about the setup can be found in [19].

We gradually increased the 1950 -nm pump power until the intensity in the T-PCF1 waist reached about $6.5 \mathrm{MW} / \mathrm{cm}^{2}$. At this intensity, the transmitted light abruptly dropped to zero, and the pump laser was shut down within two seconds. Inspection with the top-view microscope at the input side showed an input lensed fiber coated by $\mathrm{ChG}$ and the formation of a smooth ChG ball on the T-PCF1 input as shown in Fig. 2(a). Top-view inspection of the output side revealed no noticeable changes, indicating that the damage propagated backwards from an ignition point all the way back to the input where the core material blasted out. In Fig. 2(b), the SEM image shows a sphere on the T-PCF1 input. The "crater" on the

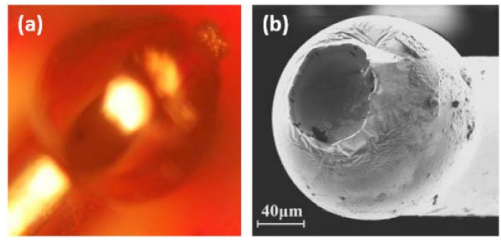

Fig. 2. (a) ChG sphere on the PCF input facet. Note the high reflection from the sphere surface. (b) SEM image of (a); the "crater" came from collision with the lensed fiber.

sphere results from the collision with the input lensed fiber during the sphere formation. The formation of such a sphere is an indication of a fast thermal equilibrium process and a discrete blast of ChG. Noticeably, a core material blast from the input facet is one of fiber fuse's distinct features, in contrast with other fiber damage mechanisms [1,2].

The rings of the air holes inside the T-PCF1 cladding prohibit us from a direct inspection of the core voids' geometries. One way to solve this issue is to cleave piecewise along T-PCF1 and image the facets. Given a proper cleaving step size, such a method could also guide us close to the starting point of fiber fuse. Due to the large number of cleaves required, the optimal approach is to start with an optical microscope and then to move the most important samples to an SEM for better details. During the process, we noticed that voids exist in both the taper waist and the input un-tapered region. The optical images of three consecutive cleaved facets inside the waist region are shown in Figs. 3(a)-3(c), respectively. Apparently, these voids in waist core have various geometries, and they are isolated. The smooth voids confined in the fiber core imply that the hot point/damage must propagate faster than the thermal conduction speed in GeAsSe. Fiber fuse also managed to travel through the input un-tapered region [Fig. 3(d)]. Thus, while the fuse ignited at $6.5 \mathrm{MW} / \mathrm{cm}^{2}$ in T-PCF1, its propagation was maintained after the intensity dropped to $1 \mathrm{MW} / \mathrm{cm}^{2}$. Facet damage from fiber fuse differs significantly from purely thermally induced facet damage, as shown in Fig. 3(e). For comparison, an intact fiber facet is included in Fig. 3(f).

Voids were observed along the whole length of T-PCF1 up until the transition region at the output side. The intact, output un-tapered region indicates that fiber fuse in T-PCF1 started at some point within the output transition region. We imaged a facet at this region with a Zeiss Merlin SEM and observed a clear footprint of fiber fuse [Fig. 4(a)]. A facet from the input

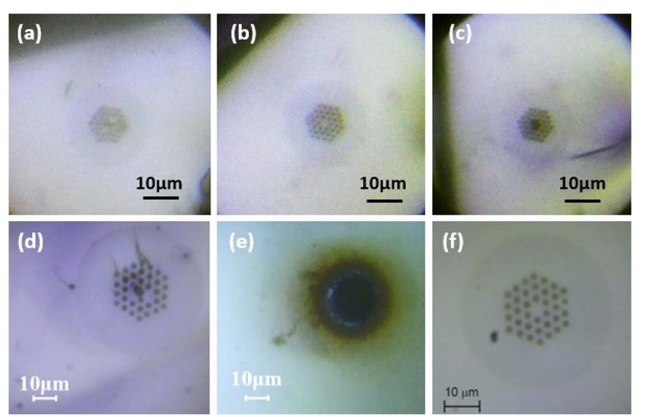

Fig. 3. (a)-(c) Optical image of waist facets after fiber fuse on various locations. (d) Core void due to fiber fuse. (e) Purely thermal induced damage on input facet. (f) Intact facet. 

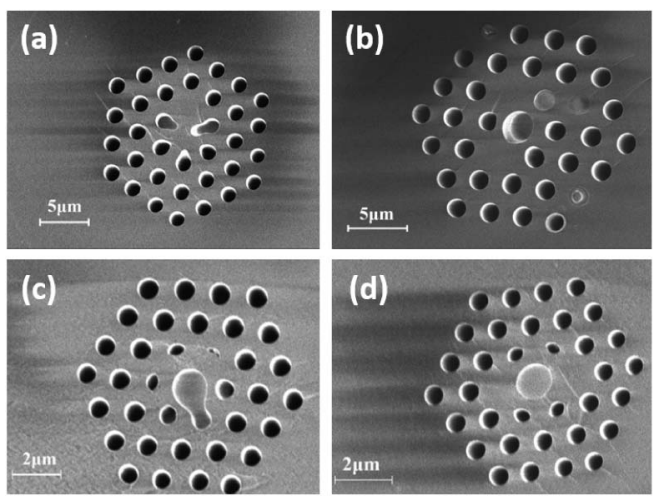

Fig. 4. (a) SEM image of a facet on output transition region of taper 1, close to the start point of fiber fuse; (b) SEM image of the facet from Fig. 3(d); (c) and (d) SEM image of two facets inside the taper waist; shallow bottoms can be seen by using the in-lens detector.

un-tapered region was also imaged, as shown in Fig. 4(b), corresponding to the facet shown in Fig. 3(d). The SEM in-lens detector allows us to check the existence of the voids' bottoms. In Figs. 4(c) and 4(d), facets from the taper waist were recorded together with the voids' bottoms. Like fiber fuse in silica, voids in GeAsSe PCF are isolated. In Fig. 4, it can be noticed that some air holes were filled by re-solidification of vaporized ChG [particularly in Figs. 4(b) and 4(d)] while the fuse voids are well confined within the core region. After the formation of the voids, high-temperature ChG managed to burn its way into the air holes, propagate a certain length, and then re-solidify along the holes during the propagation.

Rather than examining T-PCF1 with near- or mid-infrared light, "opening" the fiber is the most reliable way to check the voids' period and fuse impact on the air holes. Due to the small dimension of the taper waist, polishing can only be performed on the input un-tapered segment. A 6-mm segment of the untapered region was glued onto a holder, and the whole polishing setup is illustrated in Fig. 5(a). This holder assembly was mounted on a vertical linear translation stage. Polishing films have grain sizes ranging from $6 \mu \mathrm{m}$ to $0.02 \mu \mathrm{m}$. We monitored the polishing process in real time. The polishing angle is indicated in Fig. 5(b).

We imaged the top view of the polished segment with a $100 \times$-long working distance objective. Figure 5(c) is composed of five successive images. A fiber core is placed in the middle of the image, and the isolated voids from fiber fuse are well confined to the core. The voids period/interval is around $60 \mu \mathrm{m}$. Each period contains three smaller voids, which has a total

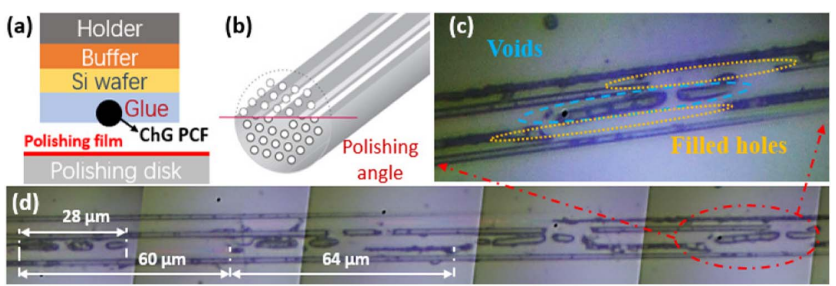

Fig. 5. (a) Sketch of the polishing assembly. (b) Polishing angle of the segment. (c) Composed image of the "opened" fiber. Note the clear periodicity of voids. (d) Enlarged image of voids' top-view.
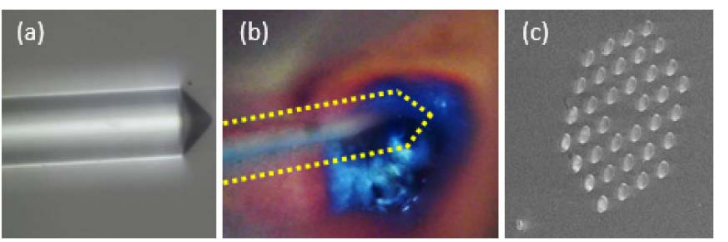

Fig. 6. (a) Input lens fiber before fuse happened. (b) Input lensed fiber coated by ChG after fiber fuse; lensed fiber is out of focus to better image the deposited ChG. (c) SEM image of the input facet of T-PCF2, showing no trace of damage on this facet.

length of $28 \mu \mathrm{m}$. Zooming in on Fig. 5(d), one can also notice the filled air holes closest to the voids. We would also like to emphasize that the images in Fig. 5 came from the un-tapered region, where the light intensity is about $1 \mathrm{MW} / \mathrm{cm}^{2}$. Since the intensity in the taper waist of the T-PCF1 region is 6.5 $\mathrm{MW} / \mathrm{cm}^{2}$, the void sizes and period/intervals can be different.

T-PCF2, tapered from the same spool as T-PCF1, has a waist length of $1.2 \mathrm{~m}$. Fiber fuse happened at a waist power intensity of $7.5 \mathrm{MW} / \mathrm{cm}^{2}$. On the input coupling side [from Figs. 6(a) and 6(b)], we observed once again that ChG had been deposited on the lensed fiber tip. However, the T-PCF2 input facet showed no noticeable damage. Further investigation confirmed that we had stopped fiber fuse at roughly $35 \mathrm{~cm}$ from TPCF2's input, with $28 \mathrm{~cm}$ of waist still transmitting light. We could estimate that fiber fuse in T-PCF2 had a minimal propagation speed of $0.5 \mathrm{~m} / \mathrm{s}$, which is in the same order of magnitude as in silica fibers [2], and orders of magnitude higher than the reported thermal damage speed in the AsS fiber [11].

Although this $28-\mathrm{cm}$ piece showed the same propagation loss as the original GeAsSe T-PCF2 $(0.5 \mathrm{~dB} / \mathrm{m})$, the large amount of deposited $\mathrm{ChG}$ on the input lensed fiber does raise concerns about its properties. As $\mathrm{ChG}$ vapor made its way to the input lensed fiber, it could have condensed and deposited on the walls of the air holes, hence effectively reducing the holepitch ratio-an effect that would also apply to silica PCF. However, to our knowledge, fiber fuse's impact on properties of remained/survived PCF has yet to be reported.

As the hole-pitch ratio directly impacts the dispersion of the PCF, dispersion measurements can provide quantitative confirmation of structural modifications. Due to the high nonlinearity and single-mode property of T-PCF2, a degenerate fourwave-mixing (DFWM) experiment can retrieve the dispersion of this segment. The phase mismatch of a DFMW process inside an optical fiber is

$$
\Delta k+2 \gamma P \approx \beta_{2} \Delta \omega^{2}+\frac{1}{12} \beta_{4} \Delta \omega^{4}+2 \gamma P=0 .
$$

In Eq. (1), $\Delta k$ is the linear phase mismatch, $\gamma$ is the nonlinear parameter of the fiber, $P$ is the coupled pump power, $\Delta \omega$ is the angular frequency difference between signal and pump, and $\beta_{2}$ and $\beta_{4}$ are the second and fourth order Taylor expansion terms of propagation constant, respectively. From a previous experiment [22], the higher order terms can be neglected for a pump wavelength less than $2.1 \mu \mathrm{m}$. Due to the small pump-signal detuning, the low propagation loss, the low pump power (roughly $5 \mathrm{~mW}$ coupled), and the short fiber length, we can safely neglect the loss and pump depletion, leading to the conversion efficiency (CE) as below: 

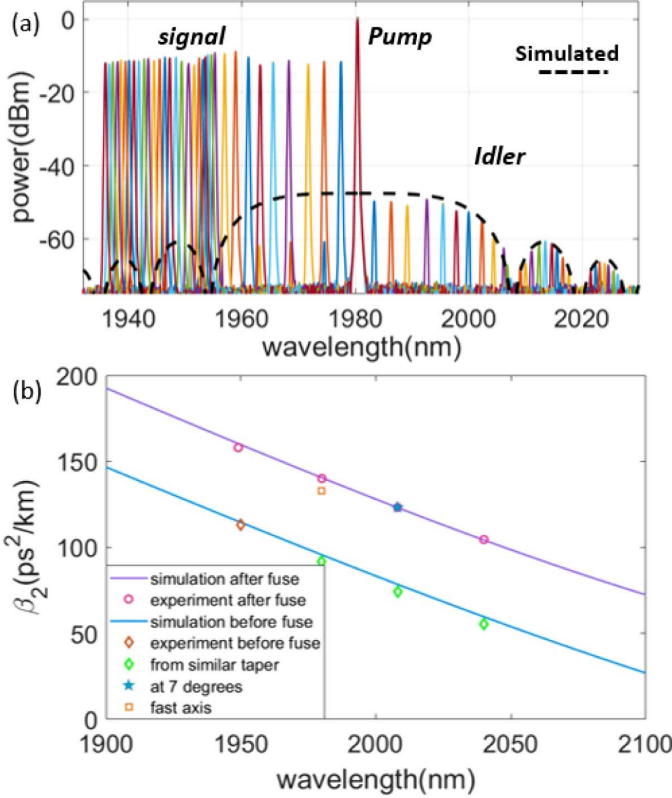

Fig. 7. (a) Superimposed spectrum of signal sweep with theoretical fitting from Eq. (2), about 5-mW pump and 0.3-mW signal power coupled into fiber. (b) Comparison of dispersion before and after fuse.

$$
\mathrm{CE}=\left(\frac{\gamma P}{\Delta k / 2}\right)^{2} \sinh ^{2}(\Delta k L / 2),
$$

wherein $L$ is the length of taper $(28 \mathrm{~cm})$. Repeatedly recording DFWM spectra at different pump wavelengths and applying the fitting (2) for the CE curves, we can retrieve the dispersion of the taper waist at various wavelengths. We simulated the dispersion of the GeAsSe PCF with different hole-pitch ratios at the $1.5-\mu \mathrm{m}$ core size using COMSOL. An interferometry measurement on the taper waist dispersion confirms the precision of our simulation [23].

In Fig. 7(a), we show the measured DFWM trace for a 1980 -nm pump in the 28 -cm segment. Clear dips appeared on the recorded spectrum, indicating good fiber uniformity and low loss. The CE curve matches perfectly with the simulation of a 0.56-hole-pitch ratio taper. Measurements with several different pump wavelengths [Fig. $7(\mathrm{~b})]$ confirms the estimated hole-pitch ratio.

The same experiment has also been performed on T-PCF2 before fiber fuse with a $1950-\mathrm{nm}$ pump. The calculated dispersion corresponded to a hole-pitch ratio of 0.58 . In Fig. 7(b), we included measured data from another taper of identical original geometry and its simulated dispersion for better comparison. The GVD difference before and after fiber fuse is significant. To exclude influences of temperature and birefringence, we performed two extra tests. Parametric conversion performed at a room temperature of $7^{\circ} \mathrm{C}$ showed no change on retrieved dispersion. The rotation of the input lens fiber showed a hole-pitch ratio variation within $2 \%$, in accordance with the previous measurement [19]. Thus, we conclude that the ChG vapor that traveled through the air holes was deposited on their walls, effectively shrinking the size of the air hole.

In conclusion, we observed and confirmed, to the best of our knowledge, the first fiber fuse inside non-silica glass fibers. This first observation could assist in designing safer MIR fiber systems. Fiber fuse on an entirely different platform with a much lower melting point can lead to a better understanding of its mechanism. However, further study must be planned and conducted with great care due to the high toxicity of $\mathrm{ChG}$. We also studied the remaining segment from T-PCF2, confirming PCF structural modifications even though fiber fuse was terminated long before this point. On the other hand, it is worth mentioning that one of our tapered GeAsSe PCF from the same spool of T-PCF1 and T-PCF2 was tested under $12 \mathrm{MW} / \mathrm{cm}^{2}$ for more than $1 \mathrm{~h}$ with no damage, limited only by our pump power. Thus, we believe a better coating process and improved fabrication can further push the power handling capability. Increasing the size of the air hole is another way to suppress the propagation of fiber fuse and can shift the zero dispersion point to better match the wavelength of $2-\mu \mathrm{m}$ fiber lasers.

Funding. H2020 European Research Council (ERC) (ERC-2012-StG 306630-MATISSE).

Acknowledgment. We thank Dr. Adrien Billat for the SEM images of the fibers and the EPFL Laboratory of Quantum Optoelectronics for lending the 100x-long working distance objective.

\section{REFERENCES}

1. R. Kashyap, Opt. Express 21, 6422 (2013).

2. S. Todoroki, Fiber Fuse (Springer, 2014).

3. D. P. Hand and P. St.J. Russell, Opt. Lett. 13, 767 (1988).

4. S. Todoroki, Opt. Express 13, 6381 (2005).

5. R. M. Atkins, P. G. Simpkins, and A. D. Yablon, Opt. Lett. 28, 974 (2003).

6. S. Jiang, L. Ma, X. Fan, S. Wang, and Z. He, Opt. Lett. 42, 3355 (2017).

7. N. Hanzawa, K. Kurokawa, K. Tsujikawa, T. Matsui, K. Nakajima, S. Tomita, and M. Tsubokawa, J. Lightwave Technol. 28, 2115 (2010).

8. W. Ha, Y. Jeong, and K. Oh, Opt. Lett. 36, 1536 (2011).

9. K. S. Abedin, M. Nakazawa, and T. Miyazaki, Opt. Express 17, 6525 (2009).

10. Y. Mizuno, N. Hayashi, H. Tanaka, K. Nakamura, and S.-I. Todoroki, Appl. Phys. Lett. 104, 043302 (2014).

11. E. Dianov, I. Bufetov, A. Frolov, V. Mashinsky, V. Plotnichenko, M. Churbanov, and G. Snopatin, Electron. Lett. 38, 783 (2002).

12. E. Dianov, I. Bufetov, A. Frolov, V. Plotnichenko, V. Mashinskii, M. Churbanov, and G. Snopatin, Quantum Electron. 32, 476 (2002).

13. G. Tao, H. Ebendorff-Heidepriem, A. M. Stolyarov, S. Danto, J. V. Badding, Y. Fink, J. Ballato, and A. F. Abouraddy, Adv. Opt. Photon. 7, 379 (2015).

14. L. Liu, T. Cheng, K. Nagasaka, H. Tong, G. Qin, T. Suzuki, and Y. Ohishi, Opt. Lett. 41, 392 (2016).

15. C.-S. Brès, S. Zlatanovic, A. O. J. Wiberg, and S. Radic, Opt. Express 19. B621 (2011).

16. N. Abdukerim, L. Li, and M. Rochette, Opt. Lett. 41, 4364 (2016).

17. L. Li, N. Abdukerim, and M. Rochette, Opt. Lett. 42, 639 (2017).

18. D. D. Hudson, S. Antipov, L. Li, I. Alamgir, T. Hu, M. E. Amraoui, Y. Messaddeq, M. Rochette, S. D. Jackson, and A. Fuerbach, Optica 4, 1163 (2017).

19. S. Xing, D. Grassani, S. Kharitonov, L. Brilland, C. Caillaud, J. Trolès, and C.-S. Brès, Optica 4, 643 (2017).

20. A. Sincore, J. Cook, F. Tan, A. El Halawany, A. Riggins, L. Shah, A. Abouraddy, M. C. Richardson, and K. L. Schepler, Laser Congress (Optical Society of America, 2017), paper ATu5A.5.

21. R. Thapa, R. R. Gattass, V. Nguyen, G. Chin, D. Gibson, W. Kim, L. B. Shaw, and J. S. Sanghera, Opt. Lett. 40, 5074 (2015).

22. S. Xing, D. Grassani, S. Kharitonov, A. Billat, and C.-S. Brès, Opt. Express 24, 9741 (2016).

23. S. Kharitonov, S. Xing, and C.-S. Brès, Laser Congress (Optical Society of America, 2017), paper JM5A.10. 